\title{
In Vivo Magnetic Resonance Microimaging of Individual Amyloid Plaques in Alzheimer's Transgenic Mice
}

\author{
Clifford R. Jack Jr, ${ }^{1}$ Thomas M. Wengenack, ${ }^{2}$ Denise A. Reyes, ${ }^{1}$ Michael Garwood, ${ }^{3}$ Geoffrey L. Curran, ${ }^{2}$ \\ Bret J. Borowski, ${ }^{1}$ Joseph Lin, ${ }^{3}$ Gregory M. Preboske, ${ }^{1}$ Silvina S. Holasek, ${ }^{2}$ Gregor Adriany, ${ }^{3}$ and Joseph F. Poduslo ${ }^{2}$ \\ ${ }^{1}$ Department of Radiology and ${ }^{2}$ Molecular Neurobiology Laboratory, Departments of Neurology, Neuroscience, and Biochemistry/Molecular Biology, Mayo \\ Clinic College of Medicine, Rochester, Minnesota 55905, and ${ }^{3}$ Center for Magnetic Resonance Research and Department of Radiology, University of \\ Minnesota Medical School, Minneapolis, Minnesota 55455
}

The ability to detect individual Alzheimer's amyloid plaques in vivo by magnetic resonance microimaging (MRI) should improve diagnosis and also accelerate discovery of effective therapeutic agents for Alzheimer's disease (AD). Here, we perform in vivo and ex vivo MRI on double transgenic AD mice as well as wild-type mice at varying ages and correlate these with thioflavin-S and iron staining histology. Quantitative counts of individual plaques on MRI increase with age and correlate with histologically determined plaque burden. Plaques $20 \mu \mathrm{m}$ in diameter can be detected in $\mathrm{AD}$ mice as young as 3 months of age with ex vivo MRI. Plaques $35 \mu \mathrm{m}$ in diameter can be detected by 9 months of age with in vivo MRI. In vivo MRI of individual Alzheimer's amyloid plaques provides a noninvasive estimate of plaque burden in transgenic AD mice that might be useful in assessing the efficacy of amyloid reduction therapies.

Key words: aging; amyloid; Alzheimer's disease; magnetic resonance microimaging; plaque; transgenic mice

\section{Introduction}

One of the cardinal pathological features of Alzheimer's disease (AD) is formation of senile, or amyloid, plaques (Hardy and Selkoe, 2002). Murine models of AD have been created by inserting human mutations leading to familial AD into the mouse genome, allowing controlled study of amyloid plaque biology (Hsiao et al., 1996; Holcomb et al., 1998). Doubly transgenic [amyloid precursor protein (APP)/presenilin 1 (PS1)] mice develop extensive "human-like" plaque formation at an early age (10 weeks), whereas plaques are not found in wild-type mice. Plaque number and size increase with age in a stereotypical manner in these transgenic AD mice with relatively low inter-animal variation in this pattern (Wengenack et al., 2000b).

Direct imaging of amyloid plaques in the brain is feasible. Scintigraphic probes label plaques in human AD postmortem tissue sections as well as in living AD subjects (Skovronsky et al., 2000; Wengenack et al., 2000a; Shoghi-Jadid et al., 2002; Bacskai et al., 2003; Klunk et al., 2004). Several groups have also pursued imaging of amyloid plaques with magnetic resonance microimaging (MRI) (Beneviste et al., 1999; Dhenain et al., 2002; Poduslo et al., 2002; Wadghiri et al., 2003; Helpern et al., 2004; Jack et al., 2004; Zhang et al., 2004; Higuchi et al., 2005). A motivation for this effort is that, unlike other modalities, MRI can resolve indi-

Received June 23, 2005; revised Aug. 23, 2005; accepted Sept. 20, 2005.

This work was supported by National Institutes of Health Grants AG22034 and RR08079, the W. M. Keck Foundation, the Mind Institute, and the Minnesota Partnership for Biotechnology and Medical Genomics. We thank Dawn M. Gregor, for mouse breeding and genotyping, Stephen Weigand and Dr. Peter C. O'Brien, for statistical assistance, and Dr. Karen Duff for the PS1 transgenic mouse line.

Correspondence should be addressed to Dr. Clifford R. Jack Jr, Diagnostic Radiology, Mayo Clinic, 200 First Street SW, Rochester, MN 55905. E-mail: jack.clifford@mayo.edu.

DOI:10.1523/JNEUROSCI.2588-05.2005

Copyright $\odot 2005$ Society for Neuroscience $\quad 0270-6474 / 05 / 2510041-08 \$ 15.00 / 0$ vidual plaques noninvasively. The work in MRI has taken several directions. One is imaging of plaques in ex vivo human or transgenic mouse specimens, typically with long imaging times that may exceed 10 h (Beneviste et al., 1999; Dhenain et al., 2002; Poduslo et al., 2002; Helpern et al., 2004; Lee et al., 2004; Zhang et al., 2004). Another is imaging of plaques in AD mice after administration of exogenous plaque-labeling contrast media (Poduslo et al., 2002; Wadghiri et al., 2003; Higuchi et al., 2005). The final direction is MRI of plaques without exogenous contrast media in the living AD mouse (Jack et al., 2004; Vanhoutte et al., 2005). The basis for intrinsic MRI contrast (i.e., without administration of an exogenous labeling agent) between individual plaques and normal background tissue is presumed to be related to the iron content of plaques, which accelerates $\mathrm{T} 2$ and $\mathrm{T} 2{ }^{\star}$ relaxation rates of tissue water protons in and adjacent to plaques (Beneviste et al., 1999).

These early efforts at establishing the ability of MRI to visualize plaques are essential steps in verifying the feasibility of this technique. However, to use this approach to address biologically interesting questions, appropriate correlations with the known biology of plaque development must be demonstrated. To this end, the broad objective of this study was to validate in vivo MRI of individual plaques as a noninvasive estimate of plaque burden in transgenic $\mathrm{AD}$ mice.

\section{Materials and Methods}

We addressed the following questions in this study: (1) Do in vivo and ex vivo MRI quantitatively capture the known age-dependent increase in plaque burden? (2) Does plaque burden quantified by in vivo and ex vivo MRI correlate with a histological gold-standard measure, thioflavin-S (Thio-S)? (3) Does the appearance of individual plaques on in vivo and ex vivo MRI correlate qualitatively with Thio-S and iron staining histology? (4) What is the youngest age at which plaques can be detected by in vivo 


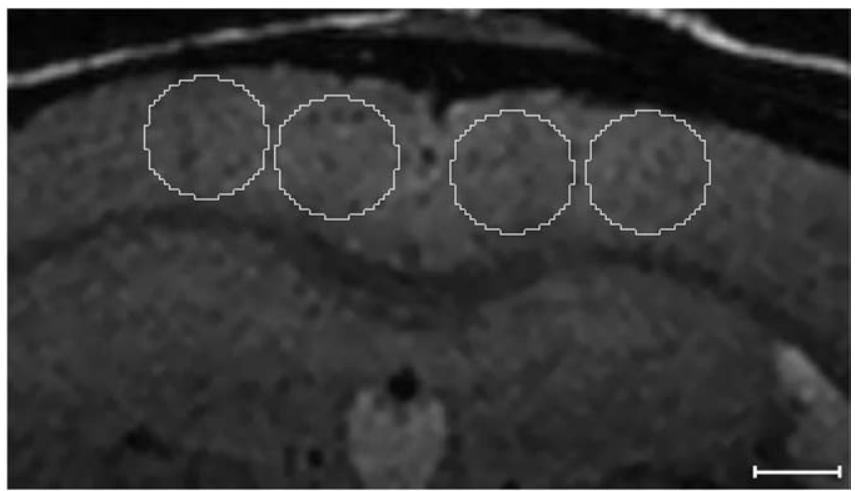

Figure 1. ROls used for plaque counting. See Materials and Methods for details. Scale bar, $500 \mu \mathrm{m}$.

and ex vivo MRI in this $\mathrm{AD}$ mouse model? (5) What are the smallest size $\mathrm{AD}$ plaques that can be resolved by in vivo and ex vivo MRI?

Imaging methods. Experiments were performed using both APP/PS1 transgenic $\mathrm{AD}$ and $\mathrm{B} 6 \mathrm{SJL}$ wild-type mice. Mice were anesthetized using $1.0-1.5 \%$ isoflurane and $\mathrm{O}_{2} / \mathrm{N}_{2} \mathrm{O}$ and positioned in a custom-built cradle for imaging. The mouse was positioned in the holder with its abdomen/thorax on top of a respiration pressure transducer of an MRIcompatible electrocardiogram and respiratory monitoring device (SA Instruments, Stony Brook, NY). The same unit also controlled the cardiorespiratory gating that triggered the scanner. The electrocardiogram was monitored via disposable adhesive electrodes affixed to the left forelimb and right hindlimb. Body temperature was monitored by a rectal probe and maintained with a hot-water circulating system. Experimental protocols were approved by the Institutional Animal Care and Use Committees of both the University of Minnesota and the Mayo Clinic in accordance with the National Institutes of Health Guide for the Care and Use of Laboratory Animals.

$\mathrm{AD}$ and wild-type mice at ages $3,6,9,12$, and 24 months were imaged in vivo with a previously described T2-weighted spin-echo sequence specifically designed for this application (Jack et al., 2004). The imaging parameters were as follows: repetition time, $2 \mathrm{~s}$; echo time, $52 \mathrm{~ms}$; $x, y, z$ matrix, $256 \times 96 \times 32$ at corresponding fields of view of $15.36 \times 5.76 \times 3.84 \mathrm{~mm}$ resulting in voxel dimensions of $60 \times 60 \times 120 \mu \mathrm{m}$, respectively, and a scan time of $1 \mathrm{~h}, 40 \mathrm{~min}$. The images were reconstructed with in-plane, twodimensional zero-padding to produce final voxel dimensions of $30 \times 30 \times 120 \mu \mathrm{m}$. MR images were filtered to smooth noise while preserving edges. Experiments were performed with a Unity Inova spectometer (Varian, Palo Alto, CA), which was interfaced to a $9.4 \mathrm{~T} / 31$ $\mathrm{cm}$ horizontal bore magnet equipped with actively shielded gradients capable of $300 \mathrm{mT} / \mathrm{m}$ in a rise time of $500 \mu \mathrm{s}$ (Magnex Scientific, Abingdon, UK). Radiofrequency (RF) transmission and reception was performed with an in-house-built quadrature surface coil consisting of two 1-cm-diameter loops.

Animal and brain tissue handling. After in vivo MRI, the animals were killed to perform correlations with ex vivo MRI and in vitro histology. These animals were perfused with PBS and fixed with neutral buffered $10 \%$ formalin after an overdose with sodium pentobarbital ( $200 \mathrm{mg} / \mathrm{kg}$, i.p.). The brain was removed, fixed further in formalin overnight, and equilibrated in 0.1 M sodium phosphate, $\mathrm{pH} 7.4$, for $24 \mathrm{~h}$. The brain was embedded vertically in $2 \%$ agar in a $15-\mathrm{mm}$-outer-diameter glass tube for ex vivo MRI. The fixed brains were imaged ex vivo with the same imaging sequence described above.

After cryoprotecting in 10\% sucrose, followed by $30 \%$ sucrose in $0.1 \mathrm{M}$ phosphate, $\mathrm{pH} 7.4$, frozen coronal sections $(30 \mu \mathrm{m})$ were cut with a sliding microtome throughout the entire extent of the cerebral cortex. The brains were sectioned in the same coronal orientation histologically as in the MRI scan. Adjacent $30 \mu \mathrm{m}$ sections were stained for Thio-S and iron. The sections were mounted on slides, dried, and stained with fresh, filtered, aqueous $1 \%$ Thio-S. The Thio-S-positive $\beta$-amyloid plaques were visualized with a fluorescence microscope using filters for fluorescein isothiocyanate.

To visualize tissue iron, a diaminobenzidine (DAB)-enhanced method of the Prussian Blue reaction was performed, as described previously (LeVine, 1991), that is sensitive enough to detect trace amounts of iron in Alzheimer's plaques (LeVine, 1997). Briefly, mounted $30 \mu \mathrm{m}$ sections were hydrated in PBS and incubated in $10 \mathrm{mg} / \mathrm{ml}$ sodium borohydride (Sigma, St. Louis, MO) in PBS for $30 \mathrm{~min}$. The sections were washed between each step in PBS twice for 5 min each. The sections were incubated for $20 \mathrm{~min}$ in $30 \mu \mathrm{g} / \mathrm{ml}$ proteinase K (Sigma) and $0.1 \%$ Triton $\mathrm{X}-100$ in PBS. The sections were then incubated in $1 \%$ potassium ferrocyanide (Sigma), $1 \% \mathrm{HCl}$, and $1 \%$ Triton X-100 in distilled water for 30 min. Finally, the sections were incubated in $0.5 \mathrm{mg} / \mathrm{ml} 3,3^{\prime}$ diaminobenzidine tetrahydrochloride (Sigma) and $2 \mu \mathrm{l} / \mathrm{ml}$ of $30 \%$ hy-
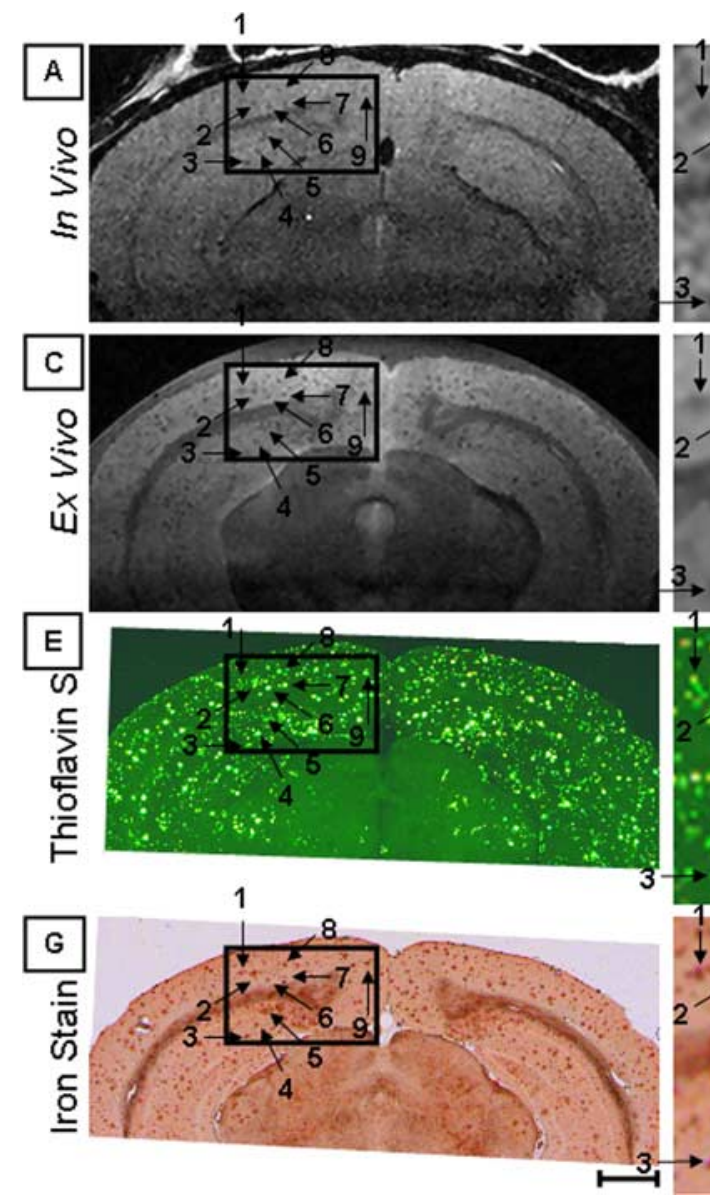

Figure 2. Twenty-four-month-old AD mouse. In vivo $\operatorname{MRI}(\boldsymbol{A}, \boldsymbol{B})$, ex vivo $\operatorname{MRI}(\boldsymbol{C}, \boldsymbol{D})$, Thio-S-stained $(\boldsymbol{E}, \boldsymbol{F})$, and iron-stained ( $\boldsymbol{G}$, $\boldsymbol{H})$ images have been precisely spatially registered over a circumscribed area of the cortex, indicated by the box. The boxes in the right column (scale bar, $100 \mu \mathrm{m}$ ) represent $3 \times$ magnified portions of the adjacent parent image in the left column (scale bar, 1.0 $\mathrm{mm}$ ). The numbered arrows indicate individual plaques visualized in each of the four different image types that matched with the linked cursor system. 

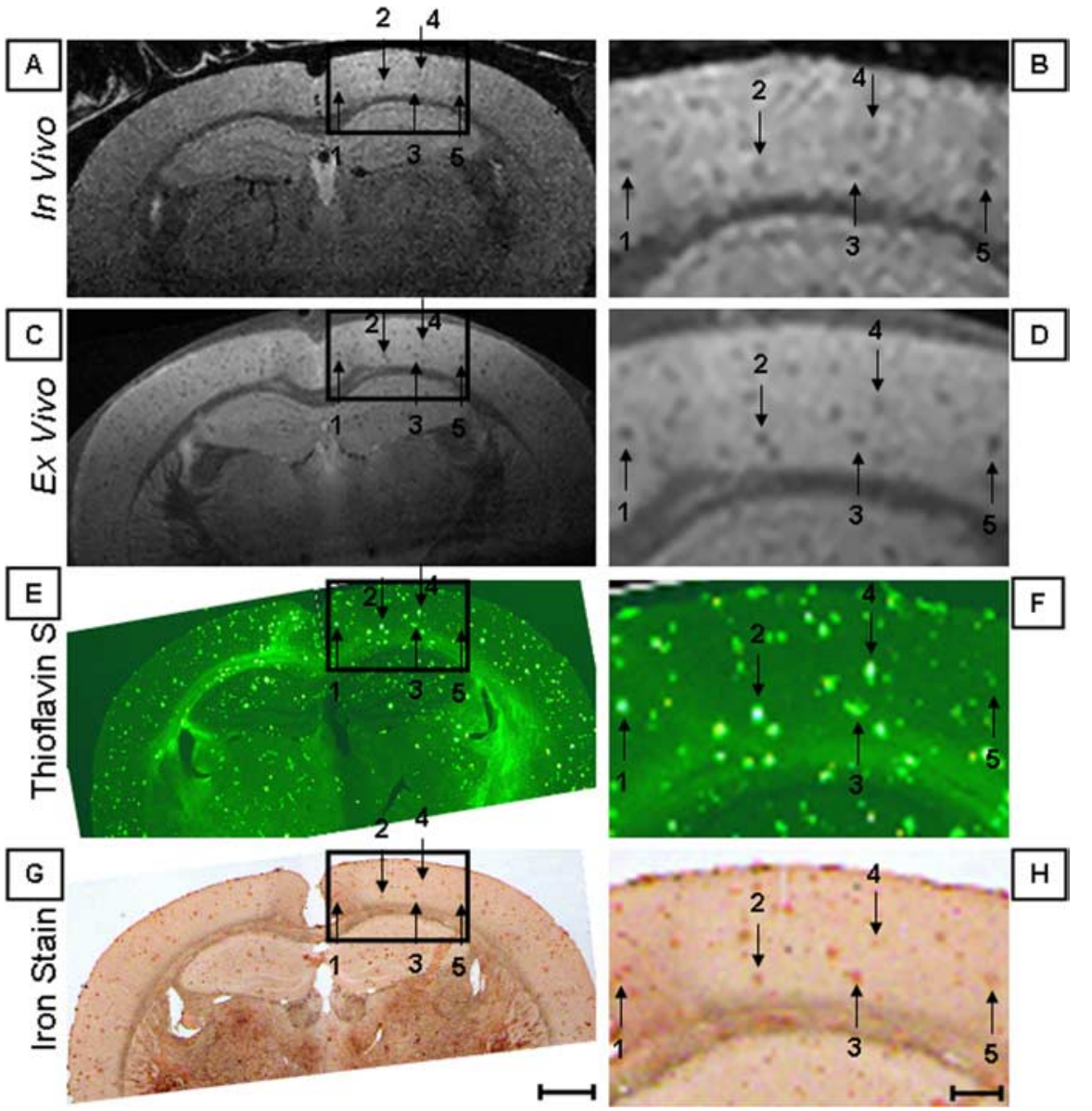

Figure 3. Twelve-month-old $A D$ mouse. In vivo $\operatorname{MRI}(\boldsymbol{A}, \boldsymbol{B})$, ex vivo $\mathrm{MRI}(\boldsymbol{C}, \boldsymbol{D})$, Thio-S-stained $(\boldsymbol{E}, \boldsymbol{F})$, and iron-stained $(\boldsymbol{G}, \boldsymbol{H})$ images have been precisely spatially registered over a circumscribed area of the cortex, indicated by the box. The boxes in the right column (scale bar, $100 \mu \mathrm{m}$ ) represent $3 \times$ magnified portions of the adjacent parent image in the left column (scale bar, $1.0 \mathrm{~mm}$ ). The numbered arrows indicate individual plaques visualized in each of the four different image types that matched with the linked cursor system.

drogen peroxide (Sigma) in $0.05 \mathrm{M}$ Tris $\mathrm{HCl}, \mathrm{pH} 7.6$, for $15 \mathrm{~min}$ in the dark. The sections were rinsed for $5 \mathrm{~min}$ in three changes of distilled water, coverslipped, and imaged without counterstaining. In negative control sections in which the potassium ferrocyanide step is omitted but the $\mathrm{DAB} / \mathrm{H}_{2} \mathrm{O}_{2}$ step is included to test for the presence of endogenous peroxidases, no plaques are stained, confirming the specificity of the staining for iron and its presence in plaques.

Qualitative MRI-histological correlation. Correlating anatomical features between in vivo and ex vivo MRI of the same mouse was accomplished using image analysis software with a linked cursor system (Robb, 1990). The ex vivo MRI volume was spatially matched to the in vivo volume using readily identifiable anatomic landmarks common to both image volumes. After affine transformation, the ex vivo MRI volume was resampled in the space of the in vivo volume with windowed sinc interpolation.

To correlate histological sections with MRI, medium resolution images of the individual histological sections were combined to create a digitized three-dimensional volume of the specimen ( 32 images per stain per mouse). Appropriate multiples of the $30 \mu \mathrm{m}$ histological sections were used to match the $120 \mu \mathrm{m}$ through-plane resolution of MRI. Adjacent $30 \mu \mathrm{m}$ sections were stained with Thio-S and DAB-enhanced Prussian Blue; therefore, matching between the MRI, Thio-S- and ironstained sections contained inherent partial volume averaging approximations. The digitized histological volume was then spatially matched to the in vivo MRI volume using anatomical landmarks common to both volumes as described above. A linked cursor system in the image analysis software program was used to identify plaques common to the four spatially registered volume data sets: in vivo MRI, ex vivo MRI, in vitro Thio-S stain, and in vitro iron stain.

Quantification methods. As outlined in the discussion, it is impossible to spatially register an entire in vivo MRI volume to an ex vivo MRI or histological volume with accuracy at the tens-of-micrometers level. The different image types were therefore globally affine registered for quantitative plaque counting. After affine registration of ex vivo MRI and Thio-S sections to the appropriate in vivo MRI sections, plaques were quantified by manual counting. Circular regions of interest (ROIs) $0.75 \mathrm{~mm}$ in diameter were placed electronically over the cortex, two ROIs per hemisphere near the midline as shown in Figure 1. This was repeated on five coronal sections evenly spaced throughout the cortex for a total 20 ROIs per image volume. The number of plaques in each ROI was counted manually. The mean number of plaques per ROI was summed for each type of image (in vivo MRI, ex vivo MRI, and Thio-S). Plaque counts were repeated four times on successive days to assess precision of the method. The SD over four repeated measures at each ROI was averaged across all plaque-containing ROIs in each animal.

The minimum plaque size resolvable on in vivo or ex vivo MRI was established by measuring plaque diameters on the corresponding Thio-S-stained sections using high-resolution photomicrographs. Areas of the cortex were selected in which individual plaques could be identified both on spatially matched in vivo (or ex vivo) MRI and Thio-S images in each of the APP/PS1 mice. The diameters of these MR visible plaques were measured on the Thio-S photomicrographs using Axiovision image analysis software (Zeiss, Thornwood, NY).

\section{Results}

Plaques seen in the cortex and hippocampus on both in vivo and ex vivo MRI appear as discrete dark foci against the brighter-intensity background of adjacent normal tissue. This is illustrated in Figure 2, which is a composite figure of four different image types in a 24-month-old AD mouse. In this image, in vivo MRI, ex vivo MRI, Thio-S-stained, and ironstained images have been precisely spatially registered over a circumscribed area of the cortex, indicated by the box. This permits accurate point-to-point correlation of individual plaques in the four different types of images. Figure 2 shows that individual plaques documented by positive Thio-S staining can be resolved with both in vivo and ex vivo MRI. It also demonstrates the correlation between iron staining of individual plaques and MRI conspicuity. The iron stain appears to label only plaques that are Thio-S positive. Figures 3-6 are similarly formed composite figures of $\mathrm{AD}$ mice aged $12,9,6$, and 3 months, respectively. A comparison of Figures $2-6$ indicates that both plaque density and size increase with the age of AD mice on histological sections and that these age-dependent features of plaque appearance are mirrored on MRI. No plaques were seen in wild-type mice at any age. A composite figure (Fig. 7) of a 9-month-old wild-type mouse demonstrates no evidence of plaques on Thio-S- or iron-stained sections and no evidence of plaques on in vivo or ex vivo MRI.

Quantification of plaque burden demonstrates that the mean 
number of plaques per ROI increases with the age of AD mice (Table 1). This is true in each of the image types evaluated quantitatively: in vivo MRI, ex vivo MRI, and Thio-S staining. Thus, plaque density on MRI scaled appropriately with the histological gold standard. Ex vivo MRI detected fewer plaques than Thio-S staining at each age, and in vivo MRI detected even fewer plaques at each age (Table 1). Taking the Thio-S plaque counts as the gold standard, the degree to which in vivo MRI underestimated true plaque burden varied with age. To calibrate MRI estimates of plaque burden with respect to the Thio-S stain, we regressed the mean plaque count per ROI at each age in MRI on the corresponding mean value in the Thio-S sections. Linear regression of in vivo MRI on Thio-S revealed a slope of 0.35 and an intercept of -2.2 (Fig. 8, filled square). This equation can be used to estimate true plaque burden (plaques per ROI in the Thio-S preparations) from in vivo MRI plaque counts. Linear regression of ex vivo MRI on Thio-S revealed a slope of 0.38 and an intercept of 0.46 (Fig. 8, open square). The reproducibility of plaque counting was best with Thio-S staining and worst with in vivo MRI. Reproducibility increased with both age and plaque density for all three image types, but this effect was most pronounced with in vivo MRI.

One benchmark we wanted to establish was the youngest age at which plaques could be reliably detected with MRI. Figures 2-6 show that the conspicuity of individual plaques is consistently superior with ex vivo compared with in vivo MR images, and as a result, the youngest age at which plaques can be resolved in vivo differs from ex vivo MRI. Plaques can be resolved at $\geq 3$ months by ex vivo MRI. Candid assessment indicates that plaques cannot be resolved from background noise on stand-alone in vivo MRI in the 3- and 6-month-old animals without the visual cues provided by other spatially registered image types. We therefore conclude that plaques can be resolved reliably by in vivo MRI at $\geq 9$ months.

Another benchmark we wanted to establish was the smallest plaque size detectable by in vivo and ex vivo MRI. The median diameters of plaques that were visible on in vivo MRI by age are shown in Figure 9A. As expected, the median diameter of MR visible plaques increases with age. The minimum plaque diameter on Thio-S that was detected by ex vivo MRI was as small as 10 $\mu \mathrm{m}$. This undoubtedly represents a partial volume effect because MR resolution was $30 \times 30 \times 120 \mu \mathrm{m}$ after two-dimensional zero-padding reconstruction, and the histological sections were $30 \mu \mathrm{m}$ thick. Because it is not possible to completely control for partial volume mismatches between the MR and Thio-S sections, a better indicator of minimum resolvable plaque size might be the median values (Fig. 9A). As indicated in above, plaques can be resolved reliably by in vivo MRI only at $\geq 9$ months. We therefore determined the median plaque diameter at 9 months, $35 \mu \mathrm{m}$, to be the minimum plaque size resolvable by in vivo MRI (Fig. 9A). Plaques could be reliably detected on ex vivo MRI at 3 months.
We therefore determined the median diameter at 3 months, 20 $\mu \mathrm{m}$, to be the minimum plaque size resolvable by ex vivo MRI (Fig. 9B).

\section{Discussion}

The major findings of this study are as follows. Quantitative counts of individual amyloid plaques on MRI increase with age in transgenic $\mathrm{AD}$ mice in a manner consistent with known pathobiology. This increase is monotonic with age on ex vivo MRI and also with in vivo MRI after a detection threshold somewhere between 6 and 9 months is reached. MRI quantification of plaque burden correlates with histologically established plaque burden in the same animals. Plaques can be resolved in APP/PS1 mice as young as 3 months with ex vivo MRI and by 9 months with in vivo MRI. Plaques histologically measured to be $35 \mu \mathrm{m}$ in diameter are detectable by in vivo MRI, and plaques $20 \mu \mathrm{m}$ in diameter are detectable by ex vivo MRI.

We were able to resolve plaques as small as $20 \mu \mathrm{m}$ ex vivo with a two-dimensional zero-padded reconstructed MRI voxel size of $30 \times 30 \times 120 \mu \mathrm{m}$. A relatively small plaque inside a larger voxel therefore must produce enough local spin dephasing, and thereby signal loss, to cause that voxel to be resolved from surrounding normal tissue. Plaques that stained with Thio-S also typically stained for iron, indicating that, as in humans, amyloid plaques in this animal model do contain iron. The source of the 

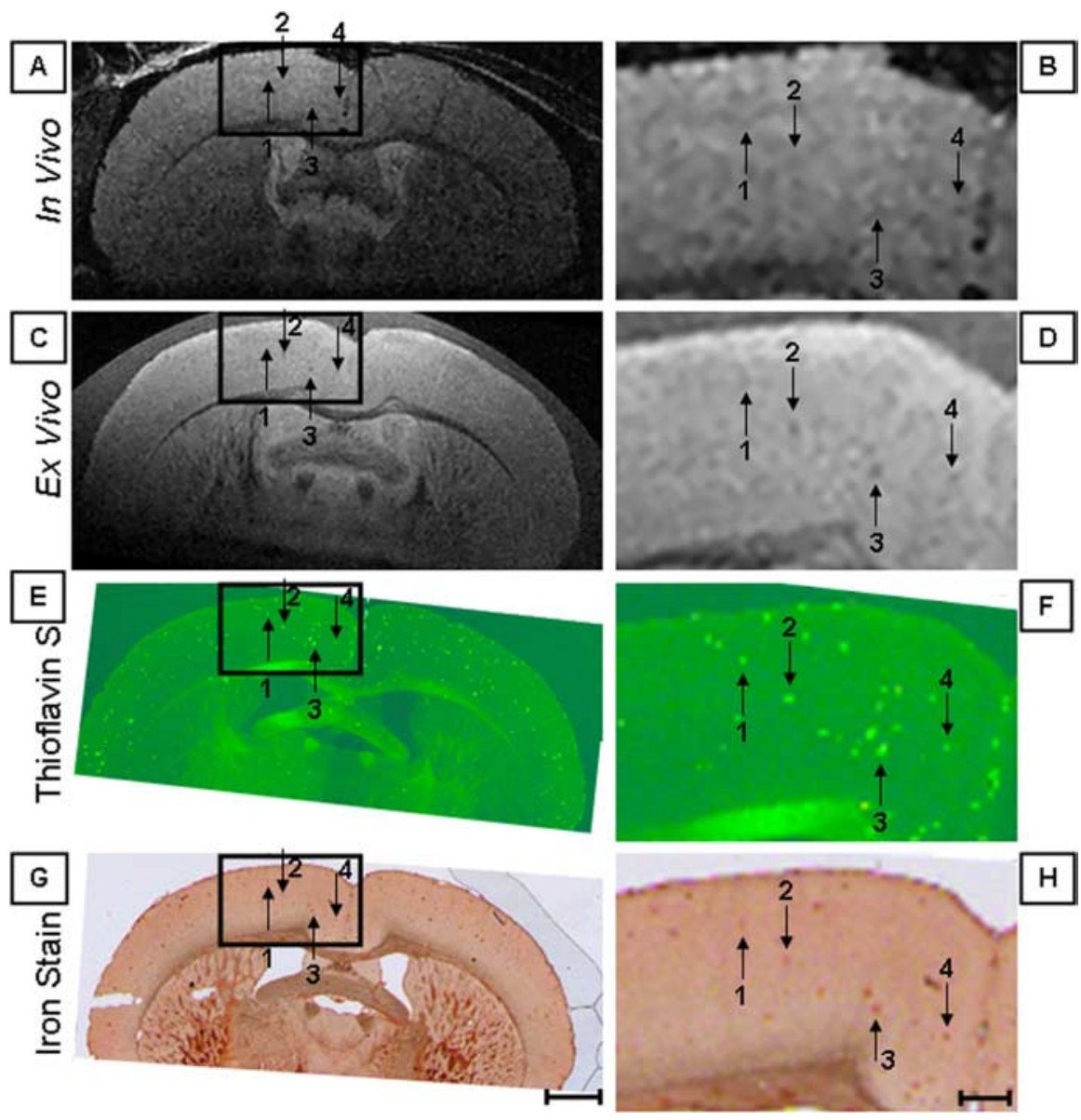

Figure 5. Six-month-old $A D$ mouse. In vivo MRI $(\boldsymbol{A}, \boldsymbol{B})$, ex vivo $M R I(\boldsymbol{C}, \boldsymbol{D})$, Thio-S-stained $(\boldsymbol{E}, \boldsymbol{F})$, and iron-stained $(\boldsymbol{G}, \boldsymbol{H})$ images have been precisely spatially registered over a circumscribed area of the cortex, indicated by the box. The boxes in the right column (scale bar, $100 \mu \mathrm{m}$ ) represent $3 \times$ magnified portions of the adjacent parent image in the left column (scale bar, $1.0 \mathrm{~mm}$ ). The numbered arrows indicate individual plaques visualized in each of the four different image types that matched with the linked cursor system.

contrast between plaques and normal-appearing adjacent brain tissue is presumably related to iron within plaques. The accelerated $\mathrm{T} 2$ relaxation observed in plaques is most likely attributable to the magnetic susceptibility effect induced by the iron itself. The faster T2 relaxation in plaques compared with normal brain could also be partially attributable to exchange between tissue water protons and protons in plaque-associated proteins. A caveat about our data on plaque diameter resolvable by MRI is that estimates of plaque size might be different had a different plaquestaining technique been used. (Styren et al., 2000)

The number of plaques per ROI seen on Thio-S exceeds the number of plaques per ROI seen on ex vivo and in vivo MRI. This is because of the fact that only plaques exceeding the 20- and $35-\mu \mathrm{m}$-diameter threshold are resolvable by ex vivo and in vivo MRI, respectively. The implication is that MRI can be considered an estimate rather than an absolute measure of plaque burden. Moreover, because the proportion of Thio-S-stained plaques $>20$ or $>35 \mu \mathrm{m}$ increases with age, the degree to which MRI underestimates Thio-S plaque burden decreases with age. Wengenack et al. (2000b) have previously quantified plaque morphology by age in this mouse model. The proportion of the total plaque load in APP/PS1 mice that exceeds the $35 \mu \mathrm{m}$ threshold for detection by in vivo MRI is $6 \%$ at 9 months, $16 \%$ at 12 months, and $20 \%$ at 24 months. As indicated above, it is not plaque size per se but rather plaque iron content that renders a plaque visible on T2 (or T2*)-weighted MRI. The contrast mechanism is not so much replacement of normal tissue in a voxel by plaque as it is achieving sufficiently different T2 relaxation in a voxel with a plaque relative to surrounding voxels containing normal tissue. Although not absolute, larger, more mature plaques tend to have higher iron content.

We estimated plaque density by MRI as the number of plaques per ROI, which is analogous to the number of plaques per high-power field in histology. We considered a stereological approach (Hyman et al., 1998) for plaque quantification with MRI but decided against it for the following reason. To improve the signal/noise ratio (SNR), our MR imaging was performed with a surface coil. This results in intrinsic non-uniformity in signal sensitivity over the field of view (i.e., the image SNR declines with increasing distance from the surface coil). With non-uniform SNR, conventional stereological plaque sampling methods would suffer a spatial bias.

Although many plaques in each section could be unequivocally matched between the histological and MR images (in vivo and ex vivo), some plaques visible in the histological sections were not visible on MRI and vice versa. In reality, perfect oneto-one correspondence between every plaque in the MRI and histological images is not possible. The histological section thickness was $30 \mu \mathrm{m}$, whereas the MRI sections were $120 \mu \mathrm{m}$ thick. Differences in partial volume averaging between MRI and histology were inevitable. Furthermore, because adjacent 30 $\mu \mathrm{m}$ histological sections were stained for Thio-S and iron, respectively, some through-plane spatial mismatching was unavoidable. In addition, the histological specimens were sliced manually. Even for a highly skilled individual, it is impossible to create sections with perfect $30 \mu \mathrm{m}$ uniformity throughout each tissue section. With MRI, however, tissue was sectioned in a rectilinear grid, and therefore some mismatch in section thickness between MRI and histological preparation was unavoidable. Perfect plaque matching without using an algorithm that introduces spatial warping was likewise not possible between in vivo and ex vivo MRI. The ex vivo MRI was performed after the brain had been removed and formalin fixed, creating nonlinear anatomic distortions.

The voxel size we selected for the MRI experiments was dictated by intrinsic tradeoffs between the SNR, spatial resolution, and imaging time in MRI. It is possible to increase the spatial resolution, SNR, or both by simply increasing the imaging time used for ex vivo MRI. However, it is not possible to increase imaging time in vivo indefinitely to meet arbitrarily established specifications for image spatial resolution and SNR. A comparison of in vivo and ex vivo MRI in Figures 2-6 indicates that in vivo MRI is clearly SNR limited. Imaging conditions were identical for in vivo and ex vivo MRI, and plaque conspicuity was always superior on ex vivo images. This may be in part attributable to shorter 
T1 relaxation times in fixed versus in vivo tissue, which would result in greater steady-state magnetization at a fixed repetition time of $2 \mathrm{~s}$ at $9.4 \mathrm{~T}$ for the former. However, the predominant reason for the discrepancy is the presence of additional sources of noise in vivo that are not present ex vivo. The major noise source is physiological, specifically fluctuations related to the cardiorespiratory cycle. Although our in vivo MRI was cardiorespiratory triggered, complete correction of view-toview cardiorespiratory variation is difficult to achieve at the present time. However, with engineering improvements in cardiorespiratory monitoring and triggering, this could change. Other strategies to improve image quality include increasing the SNR, for example with even higher field strength and an RF coil design with multiple elements or with better coupling.

Several useful applications of this approach can be envisioned in transgenic $\mathrm{AD}$ mice. The $>1 \mathrm{~h}$ imaging time we used for in vivo MRI can be repeated many times over the life span of an animal. Because individual plaques can be resolved noninvasively, in theory MRI could be used to study plaque biology longitudinally. Natural history studies can be envisioned in which the temporal and regional characteristics of plaque development are characterized. Perhaps of greater interest is using this approach to assess the effect of experimental amyloid reduction therapeutic interventions at the level of the individual plaque. One major caveat, however, relates to the fact that visualization of individual plaques on MRI (without administration of a plaque-labeling contrast agent) is attributable to relaxation effects of plaqueassociated iron. It is possible that amyloid reduction may not be associated with removal or dispersal of the iron associated with plaque. The equations we provide relating plaque load on MRI to that on Thio-S staining likewise might differ if a therapeutic approach altered the ratio of plaque iron to plaque amyloid concentration.

The demonstrated ability to visualize plaques in vivo in mice naturally raises the question of extension of the technique to humans. Although this possibility should not be summarily excluded, a number of significant technical barriers must be solved for this technique to be viable in the living human subject. First, scan times would have to be reduced by approximately one order of magnitude, and the reduction in scan time would have to be achieved with no significant loss of image SNR or spatial resolution compared with the acquisition in mice. Second, improved methods of reducing artifacts from physiological motion and correction of bulk head motion would be needed in human subjects. Finally, our data indicate that only $20 \%$ of the total plaque burden can be resolved by in vivo MRI in 24month-old APP/PS1 mice and the plaque load in APP/PS1 mice at this age significantly exceeds that found in the typical human $\mathrm{AD}$ patient (Takeuchi et al., 2000).

In summary, we have documented a correlation between in vivo and ex vivo MRI quantification of plaques and histological plaque counting in $\mathrm{AD}$ transgenic mice of various ages. In vivo and ex vivo MRI can estimate plaque burden at the resolution level of individual plaques. This study serves as a foundation for using in vivo MRI of transgenic mice (or other animal models) as a surrogate measure of plaque burden in natural history studies of plaque biology as well as drug discovery studies of Alzheimer's amyloid plaque prevention and reduction.

\section{References}

Bacskai BJ, Hickey GA, Skoch J, Kajdasz ST, Wang Y, Huang GF, Mathis CA, Klunk WE, Hyman BT (2003) Four-dimensional multiphoton imaging of brain entry, amyloid binding, and clearance of an amyloid-beta ligand in transgenic mice. Proc Natl Acad Sci USA 100:12462-12467.

Beneviste H, Einstein G, Kim KR, Hulette C, Johnson GA (1999) Detection of neuritic plaques in Alzheimer's disease by magnetic resonance microscopy. Proc Natl Acad Sci USA 96:14079-14084.

Dhenain M, Privat N, Duyckaerts C, Jacobs RE (2002) Senile plaques do not induce susceptibility effects in $\mathrm{T}^{\star}{ }^{*}$ - weighted MR microscopic images. NMR Biomed 15:197-203.

Hardy J, Selkoe DJ (2002) The amyloid hypothesis of Alzheimer's disease: progress and problems on the road to therapeutics. Science 297:353-356.

Helpern JA, Lee S-P, Falangola MF, Dyakin V, Bogart A, Ardekani B, Duff K, Branch C, Wisniewski T, de Leon MJ, Wolf O, O'Shea J, Nixon RA (2004) MRI assessment of neuropathology in a transgenic mouse model of Alzheimer's disease. Magn Reson Med 51:794-798.

Higuchi M, Iwata N, Matsuba Y, Sato K, Sasamoto K, Saido T (2005) $19 \mathrm{~F}$ and $1 \mathrm{H}$ MRI detection of amyloid beta plaques in vivo. Nat Neurosci 8:527-533. 

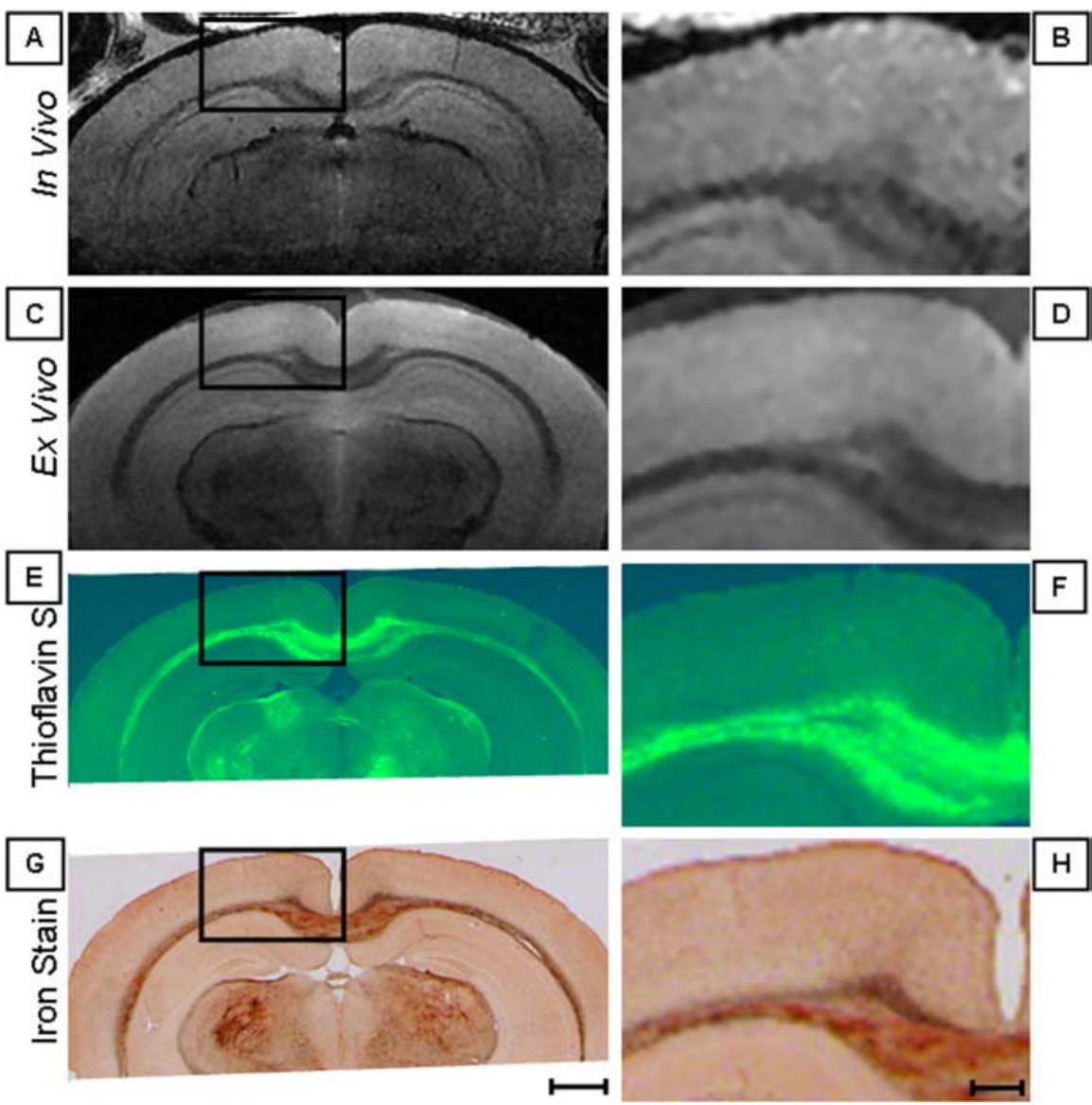

Figure 7. Nine-month-old wild-type mouse. In vivo MRI $(\boldsymbol{A}, \boldsymbol{B})$, exvivo MRI $(\boldsymbol{C}, \boldsymbol{D})$, Thio-S-stained $(\boldsymbol{E}, \boldsymbol{F})$, and iron-stained $(\boldsymbol{G}, \boldsymbol{H})$ images have been spatially registered using anatomic landmarks. The boxes in the right column (scale bar, $100 \mu \mathrm{m}$ ) represent $3 \times$ magnified portions of the adjacent parent image in the left column (scale bar, $1.0 \mathrm{~mm}$ ). Note that no plaques are visible on Thio-S or iron stains, and none are evident on the MR images.

Holcomb L, Gordon M, McGowan E, Yu X, Benkovic S, Jantzen P, Wright K, Saad I, Mueller R, Morgan D, Sanders S, Zehr C, O’Campo K, Hardy J, Prada CM, Eckman C, Younkin SG, Hsiao K, Duff K (1998) Accelerated Alzheimer-type phenotype in transgenic mice carrying both mutant amyloid precursor protein and presenilin 1 transgenes. Nat Med 4:97-100.

Hsiao K, Chapman P, Nilsen S, Eckman C, Harigaya Y, Younkin D, Yang F, Cole GM (1996) Correlative memory deficits, A-beta elevation, and amyloid plaques in transgenic mice. Science 274:99-102.

Hyman BT, Gomez-Isla T, Irizarry MC (1998) Stereology: a practical primer for neuropathy. J Neuropathol Exp Neurol 57:305-310.

Jack Jr CR, Garwood M, Wengenack TM, Borowski B, Curran GL, Lin J, Adriany G, Grohn OHJ, Grimm RC, Poduslo JF (2004) In vivo visualization of Alzheimer's amyloid plaques by MRI in transgenic mice without a contrast agent. Magn Reson Med 52:1263-1271.

Klunk WE, Engler H, Nordberg A, Wang Y, Blomqvist G, Holt DP, Bergstrom M, Savitcheva I, Huang GF, Estrada S, Ausen B, Debnath ML, Barletta J, Price JC, Sandell J, Lopresti BJ, Wall A, Koivisto P, Antoni G, Mathis CA, et al. (2004) Imaging brain amyloid in Alzheimer's disease with Pittsburgh Compound-B. Ann Neurol 55:306-319.

Lee S-P, Falangola MF, Nixon RA, Duff K, Helpern JA (2004) Visualization of amyloid plaques ina transgenic mouse model of Alzheimer's disease using MR microscopy without contrast reagents. Magn Reson Med 52:538-544.

LeVine SM (1991) Oligodendrocytes and myelin sheaths in normal, quaking, and shiverer brains are enriched in rion. J Neurosci 29:413-419.

LeVine SM (1997) Iron deposits in multiple sclerosis and Alzheimer's disease brains. Brain Res 760:298-303.

Poduslo JF, Wengenack TM, Curran GL, Wisniewski T, Sigurdsson EM, Macura SI, Borowski BJ, Jack Jr CR (2002) Molecular targeting of Alzheimer's amyloid plaques for contrast-enhanced magnetic resonance imaging. Neurobiol Dis 11:315-329.

Robb RA (1990) A software system for interactive and quantitative analysis of biomedical images. In: 3D imaging in medicine (Hohne KH, Fuchs H, Pizer SM, eds), pp 333-361. Berlin-Heidelberg: Springer.

Shoghi-Jadid K, Small GW, Agdeppa ED, Kepe V, Ercoli LM, Siddarth P, Read S, Satyamurthy N, Petric A, Huang SC, Barrio JR (2002) Localization of neurofibrillary tangles and betaamyloid plaques in the brains of living patients with Alzheimer disease. Am J Psychiatry 10:24-35.

Skovronsky DM, Zhang B, Kung M-P, Kung HF, Trojanowski JQ, Lee VM (2000) In vivo detection of amyloid plaques in a mouse model of Alzheimer's disease. Proc Natl Acad Sci USA 97:7609-7614.

Styren SD, Hamilton RL, Styren GC, Klunk WE (2000) X-34, a fluorescent derivative of Congo red: a novel histochemical stain for $\mathrm{Alz}$ heimer's disease pathology. J Histochem Cytochem 48:1223-1232.

Takeuchi A, Irizarry MC, Duff K, Saido TC, Ashe KH, Hasegawa M, Mann DVA, Hyman BT, Iwatsubo T (2000) Age-related amyloid betadeposition in transgenic mice overexpressing both Alzheimer mutant presenilin 1 and amyloid beta-precursor protein Swedish mutant is not associated with global neuronal loss. Am J Pathol 157:331-339.

Vanhoutte G, Dewachter I, Borghgraef P, Van Leuven F, Van der Linden A (2005) Noninvasive in vivo MRI detection of neuritic plaques associated with iron in APP[V717I] transgenic mice, a model for Alzheimer's disease. Magn Reson Med 53:607-613.

Wadghiri YZ, Sigurdsson EM, Sadowski M, Elliott JI, Li Y, Scholtzova H, Tang CY, Aguinaldo G, Pappolla M, Duff K, Wisniewski T, Turnbull DH (2003) Detection of Alzheimer's amyloid in transgenic mice using magnetic resonance microimaging. Magn Reson Med 50:293-302.

Wengenack TM, Curran GL, Poduslo JF (2000a) Targeting Alzheimer amyloid plaques in vivo. Nat Biotechnol 18:868-872.

Wengenack TM, Whelan S, Curran GL, Duff KE, Poduslo JF (2000b) Quantitative histological analysis of amyloid deposition in Alzheimer's double transgenic mouse brain. Neuroscience 101:939-944.

Zhang J, Yarowsky P, Gordon MN, DiCarlo G, Munireddy S, van Zijl PCM, Mori $S$ (2004) Detection of amyloid plaques in mouse models of Alzheimer's disease by magnetic resonance imaging. Magn Reson Med 51:452-457. 
Table 1. Number of plaques per ROI by age and image type

\begin{tabular}{|c|c|c|c|c|c|c|c|c|c|}
\hline \multirow[b]{2}{*}{ Age (months) } & \multicolumn{3}{|l|}{ In vivo } & \multicolumn{3}{|l|}{ Ex vivo } & \multicolumn{3}{|l|}{ Thio-S } \\
\hline & Mean & SD & $\mathrm{CV}$ & Mean & SD & $\mathrm{CV}$ & Mean & SD & $\mathrm{CV}$ \\
\hline 3 & & & & 0.83 & 0.40 & 0.48 & 4.00 & 0.30 & 0.08 \\
\hline 6 & & & & 2.38 & 0.70 & 0.29 & 7.80 & 0.50 & 0.06 \\
\hline 9 & 0.61 & 0.60 & 0.98 & 4.68 & 0.80 & 0.17 & 8.83 & 0.60 & 0.07 \\
\hline 12 & 1.46 & 0.50 & 0.34 & 7.28 & 0.80 & 0.11 & 12.04 & 0.60 & 0.05 \\
\hline 24 & 7.06 & 1.00 & 0.14 & 9.14 & 0.60 & 0.07 & 25.00 & 0.90 & 0.04 \\
\hline
\end{tabular}

Values for 3- and 6-month-old mice on in vivo MRI are not shown. CV, Coefficient of variation.

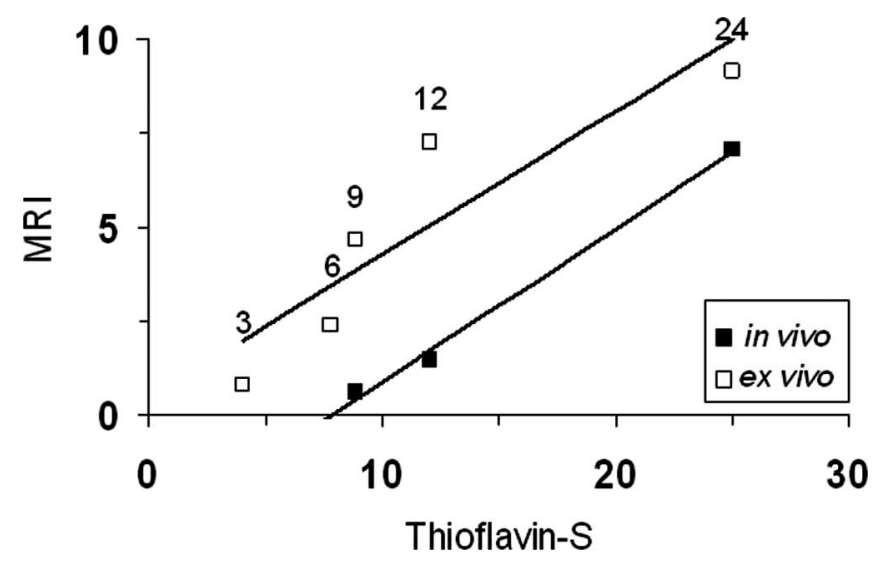

Figure 8. Mean number of plaques per ROI. MRI versus Thio-S in AD mice aged 3,6,9, 12, and 24 months.

A

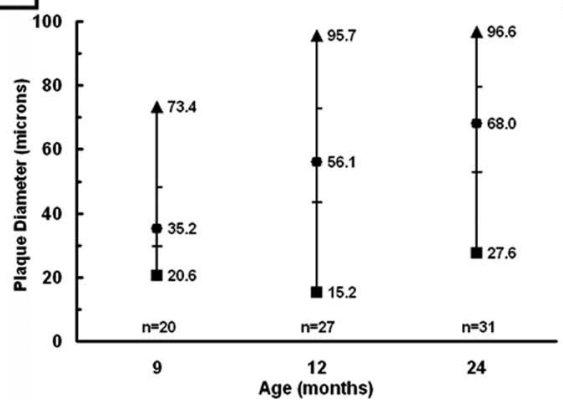

B

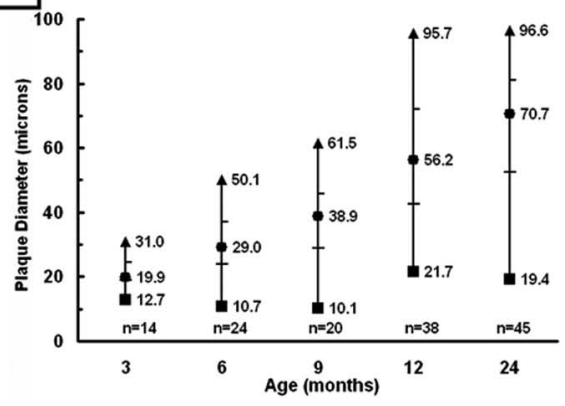

Figure 9. Diameter of plaques visible on in vivo $(\boldsymbol{A})$ and ex vivo (B) MRI by age. The ordinate plots the maximum (filled triangle), minimum (filled square), median (filled circle), 25th percentile (long dash), and 75th percentile (short dash) of the plaque diameter in micrometers measured in the corresponding Thio-S-stained histological sections. The values of each maximum, minimum, and median are noted next to each symbol. The abscissa plots the age of the APP/PS1 mice in months. The number of plaques measured for each mouse is noted above the abscissa. 